\title{
La revue Obésité, récompensée par le prix éditorial de la presse médicale et des professions de santé pour la deuxième année consécutive !
}

\author{
M. Pigeyre $\cdot$ L. Genser $\cdot$ J. Dargent \\ (C) Lavoisier SAS 2017
}

Chers lecteurs,

Nous avons le plaisir de vous annoncer que la revue Obésité a été récompensée cet automne, pour la deuxième année consécutive, par le SPEPS (Syndicat de la presse et de l'édition des professionnels de santé) et a reçu le prix du meilleur article de formation pour les médecins spécialistes pour l'article « De la dysfonction du tissu adipeux blanc aux phénotypes anatomocliniques de l'obésité » d'Olivier Ziegler, Philip Böhme et Philippe Valet. Nous en profitons pour remercier tous les auteurs, qui soumettent leurs travaux originaux, réflexions, revues, mises au point, etc., et qui enrichissent ainsi le contenu d'Obésité.

Ce dernier numéro de l'année 2017 est principalement consacré au congrès annuel de l'Association française d'étude et de recherche sur l'obésité (AFERO) qui se tiendra à Lyon les 23 et 24 janvier 2018. Ainsi, vous trouverez, le programme et les résumés des communications orales et affichées qui y seront présentées.
Nous vous invitons aussi à découvrir l'article passionnant de A. Nicolas et al. sur le rôle de la T-cadhérine en santé humaine, une revue "up-to-date » sur les obésités monogéniques par C. Bui, M. Pigeyre et D. Meyre ; puis, l'article original de C. Dumoulin, E. Reynes et S. Berthouze, dans lequel les auteurs présentent leur protocole de faisabilité pour l'amélioration durable de la pratique d'une activité physique. Jérôme Dargent continue également de nous conter l'épopée de la chirurgie bariatrique, avec un troisième volet consacré aux chirurgies restrictives. Finalement, vous retrouverez nos chroniqueurs V. Florent et B. Gaudrat pour une revue de presse sur le versant « médical » de l'obésité et nos chirurgiens, C. Blanchard, L. Genser, A. Sterkers et Ch. Barrat, pour le versant « chirurgical ».

Nous espérons que vous prendrez plaisir à lire ce numéro d'Obésité et vous souhaitons d'excellentes fêtes de fin d'année ! 